\title{
Modeling the Evaluation of Human-Vehicle Interaction with Multiple Indices
}

\author{
$\mathrm{Pu} \mathrm{Li}$, Chunming $\mathrm{Li}^{\text {a) }}$ and Zhiyou Fan \\ China North Vehicle Research Institute, Beijing, 100072, China. \\ a) Corresponding author: 550456083@qq.com
}

\begin{abstract}
We herein try to improve the human-machine interaction of military vehicles, which need the evaluation model of designing user interface (UI). Current evaluation of UI depended on the measured data of a large number of users, which were time-consuming as well as lacking quantitative evaluation. And there was a little report for evaluating UI of military vehicles. This study modeled the frequency, importance, operation sequence, correlation, uniformity and the operating path the as the evaluation indices. Also, we built the single objective function by weighting the evaluation indices and analyzed the effectiveness of our model.
\end{abstract}

Key words: Human-Vehicle Interface; Operating Path; Human-Vehicle Matching Degree; Genetic Algorithm.

\section{INTRODUCTION}

The human-vehicle interface of the tank is the carrier and medium of information interaction between driver and tank. The layout of the human-vehicle interface has an important influence on the driver's visual comfort, operation accuracy and overall combat effectiveness of equipment. Domestic and foreign scholars have conducted in-depth research on the layout design of the human-vehicle interface. In 1964, American scholar Baffa studied the layout of human-vehicle interface and put forward the relevant model. The model considered that the frequency determined the placement of the functional unit, the most frequently used functional units should be located in the optimal area. In 1993, based on Fitts's law, Errol R. Hoffmann did a research on the optimal layout of the control elements. The arrangement made up of knobs or keys would contribute to the best control size based on the minimum control time, as well as the best layout of the operating elements. The domestic research on human-vehicle interface layout model is relatively late. In 1999, Wang Ligang and Yuan Xiugan ${ }^{1}$ from Beijing University of Aeronautics and Astronautics summarized and analyzed three computer-aided design models of control panel based on ergonomics which included CRAFT model, CAPABLE model, and CSPB model. In 2006, Song Zhenghe, Mao Enrong, and Zhou Yiming ${ }^{2}$ applied the optimization design theory and method to the design of human-vehicle interface of the mechanical system. According to the design criteria of the human-vehicle interface, the mathematical model of human-vehicle interface layout optimization of mechanical system was proposed. In the aspect of layout algorithms, Jaydeep Balakrishnan, Chunhung Cheng and Kam-Faiwong proposed a heuristic FACOPT that was effective for user-friendly device layout and optimization in 2003. The software integrated simulated annealing and genetic algorithms to effectively address facility layout issues. The domestic research on the layout algorithm is late. In 1997, Fu Shibo, Yuan Xiugan ${ }^{3}$ and other fields accomplished solid modeling of meters in workplace area using CAD technology. Based on the anthropometric data, as well as in accordance with the ergonomic principles, genetic algorithm was used to optimize the layout of the work area. In 2006, Zeng Yi and Zhu Changa ${ }^{4}$ used multi-dimensional real-number coding and mapping methods to apply continuous particle swarm optimization (PSO) in the solution of the circular layout of equipment, which provided a new idea for solving such discrete optimization problems. In 2009 to 2011 , Yan Shengyuan and Wang Shanling ${ }^{5-6}$ summarized the importance, frequency, relativity and operating sequence as the principles of the layout for the human-vehicle interface in nuclear power station main control room. In this paper, the 
human-vehicle interface of nuclear power station main control room was considered as a two-dimensional space using genetic algorithm and ant colony algorithm to optimize its layout.

At present, domestic research on the human-vehicle interface of weapon system mainly focuses on the ship and aircraft and another large equipment interface. The research on the display interface of tank armored vehicles is in its infancy which mainly focus on the aspects of virtual simulation and so on. The research and design of the interactive interface are also rare. According to the principles of the shortest operation path for a crew member, functional grouping, importance, frequency, operating sequence, correlation and uniform distribution of control elements in the display interface are determined to establish the mathematical model of the layout for tank occupant interface. Then the genetic algorithm is used to solve the layout optimization objective function and seek the optimal layout optimization scheme in the search space. Finally, comparing to the optimization before, this model has better validity and feasibility, which provides a feasible method for the layout optimization of the human-vehicle interface of the tank.

\section{PROBLEM DESCRIPTION}

The research object of this paper is the interface of the tank, which can complete battlefield search, target strike, command and control, active protection and other operational tasks on the digital human-vehicle interface. According to different operation tasks, the interface can be divided into five functional groups: command and control group, task management group, battlefield search group, view attack group and parameter setting group, and then set them as five sub-interface corresponding to the control interface. Each functional sub-interface also includes the corresponding control components. The control interface optimization design is to determine the coordinates of each control element in the corresponding control interface and this article takes the command control function group as an example. The control components and their dimensions in control function group are shown in Table 1.

TABLE 1. The components information in control function group.

\begin{tabular}{|c|c|c|}
\hline Serialt Number & Part name & Part size $(\mathbf{m m} \times \mathbf{m m})$ \\
\hline 1 & Time display & $150 \times 150$ \\
\hline 2 & message notification & $1100 \times 150$ \\
\hline 3 & Navigation button & $1150 \times 150$ \\
\hline 4 & View & $1600 \times 850$ \\
\hline 5 & The car information & $450 \times 400$ \\
\hline 6 & Enemy target list & $450 \times 400$ \\
\hline 7 & Enemy target information & $450 \times 300$ \\
\hline 8 & Hand automatic targeting & $250 \times 100$ \\
\hline 10 & Loading state & $450 \times 250$ \\
\hline 11 & Ammunition margin & $350 \times 200$ \\
\hline
\end{tabular}

In order to let the layout of each control element meet the principles of ergonomics mostly, this article set the coordinate position of the control element on the control function group interface, considering six factors, which are the use of frequency, importance, operating sequence, relevance, uniformity and operating path.

\section{OPTIMIZATION MODEL}

This article chooses command and control functional group to set layout, and the interface consists of 11 control components. First, number each control unit, and the ith control component is $K_{i}$.If the center of the interface is set as the origin of coordinates, then the coordinates of the center of the $K_{i}$ control component are $\left(X_{i}, Y_{i}\right)$, and the size of the entire interface is constant as well as the size of the control components Then the position of the control components in the interface can be determined by the central coordinate positions of the control component of $K_{i}$. 


\section{The Optimization Model for Layout}

The six layout principles, usage frequency, importance, operating sequence, relevance, uniformity and operating path are quantized as six sub-objective functions $F_{i}(X, Y) \quad(i=1,2,3,4,5,6) . X=\left(X_{1}, X_{2,}, \ldots, X_{11}\right)$, $Y=\left(Y_{1}, Y_{2}, \ldots, Y_{11}\right)$. Then the six sub-objective functions are combined by weighting method to convert the multiobjective problem into a single-objective problem:

$$
\max F(X, Y)=\omega_{1} F_{1}+\omega_{2} F_{2}+\omega_{3} F_{3}+\omega_{4} F_{4}-\omega_{5} F_{5}-\omega_{6} F_{6}
$$

The bigger value of the positive sign means a better result while the number of the negative sign is on the opposite. $\omega_{i}(i=1,2,3,4,5,6)$ is the weighted value of the sub-objective function, which can be weighted according to the specific situation to adjust the impact of various layout principles.

When establishing the constraints of the interface optimization layout, there are mainly two types of them, boundary constraints and overlap constraints. The boundary constraints require the layout cannot exceed the boundaries of the interface, and the overlapping constraints require the positions of the components cannot intersect each other. Then the constraints are as follows:

$$
\left\{\begin{array}{l}
\frac{s_{i}}{2} \leq X_{i} \leq L-\frac{s_{i}}{2} \\
\frac{q_{i}}{2} \leq Y_{i} \leq L-\frac{q_{i}}{2} \\
\left|X_{i}-X_{j}\right| \geq \frac{s_{i}+s_{j}}{2} \\
\left|Y_{i}-Y_{j}\right| \geq \frac{q_{i}+q_{j}}{2}
\end{array}\right.
$$

where $L$ means the length of the interface, $W$ means the width of the interface, are the direction size in $X, Y$ of the component $i$.

\section{Sub-Objective Function Fi(X，Y)}

\section{The Importance and Frequency}

The importance and frequency of the components are the main principles that influence the layout of the interface. The prerequisite of establishing the optimized mathematical model is to quantify these layout principles as the weight of the components. In this study, fuzzy analytic hierarchy process is used to establish the fuzzy complementary matrix of the importance and frequency of components which are quantified as the weight of the layout principles. Then the weight will be considered as the restrictions of the variables in the objective function.

Firstly, establish the fuzzy complementary matrix of the manipulation elements in the command and control function group as shown in the following table. Then the frequency and the importance of the components will be ordered singly in the form of numerical value using fuzzy analytic hierarchy process to represent the weight of each component.

The formula for solving the weight of fuzzy complementary judgment matrix is as follows:

$$
Q_{i}=\frac{\sum_{j=1}^{n} a_{i j}+\frac{n}{2}-1}{n(n-1)},(i=1,2, \cdots n)
$$

Where $a_{i j}$ is the value in $i$ line, $j$ column of the matrix, $n$ is the number of components.

Conducting the consistency check for the fuzzy complementary matrix. Due to the complexity of the research 
problem and the fuzziness of cognition, the fuzzy complementary matrices are not consistent. In this case, the calculation result of the weight vector will not be reliable as a basis for decision-making. The literature ${ }^{7}$ has derived a method to test the consistency principle by using the consistency of fuzzy judgment matrix. After calculation, the above-mentioned fuzzy complementary matrices all meet the consistency requirements. Therefore, we obtain the weight value of the importance and frequency for each manipulation element in the command and control interface as shown in Table 4, where $I m_{i}$ and $F r_{i}$ stand for the importance weight as well as the frequency weight of component $i$.

The importance and frequency are directly related to the coordinates of the best viewpoints in the layout space. The greater the weight value of the importance and the frequency are, the closer the component should be to the middle of the viewing area, and the value is proportional to the distance between the component and the best viewpoint. The quantized sub-objective function based on the principles of importance and frequency are as follows:

$$
\begin{gathered}
F_{1}(\boldsymbol{X}, \boldsymbol{Y})=\sum_{i=1}^{n} \operatorname{Im}_{i} \llbracket\left(C-d_{i p}\right) \\
F_{2}(\boldsymbol{X}, \boldsymbol{Y})=\sum_{i=1}^{n} \operatorname{Fr}_{i} \llbracket\left(C-d_{i p}\right)
\end{gathered}
$$

Where $C$ is a fixed constant, and $d_{i p}$ is the distance from component $i$ to the best viewpoint $p$. The larger value for $F_{1}(\boldsymbol{X}, \boldsymbol{Y})$ and $F_{2}(\boldsymbol{X}, \boldsymbol{Y})$ will represent a better layout conforming to the principles of importance and frequency.

\section{The operating sequence}

The operation means the action based on the need of work including signal monitoring and control of components. The components that are preferentially operated during operation are supposed to be arranged in a marginalized manner according to the principle of ergonomics. Thus the component should be arranged in the edge region of the interface. However, the components in the command and control function group are required to complete multiple operating conditions, and the order of the corresponding components in each operating condition is different. In addition, some components are not used in certain operating conditions. Considering the above problems, the order of the command and control function group under multi-operating conditions can be indicated in the table below, where the figures stand for the order code of the corresponding components in a certain operating condition and 0 means that in the realization of the operating condition, this component is not used.

In order to consider the principle of operation sequence comprehensively under multi-conditions, the sequence of the operating condition is quantized into numerical values according to a certain calculation formula and the values represent the weight of each element in the operation sequence principle. This Literature describes a method for solving the weight of operating sequence in multiple operating conditions. The larger the value is, the greater the influence will be to the operating sequence, so the component should be laid preferentially near the edge of the interface. The formula for solving the coefficient of operation sequence is as follows:

$$
I_{j}=\frac{\left(\sum_{i=1}^{n} f\left(a_{i j}\right)\right)^{2}}{\sum_{i=1}^{n} a_{i j}}
$$

Where $f\left(a_{i j}\right)=\left\{\begin{array}{l}0, a_{i j}=0 \\ 1, a_{i j} \neq 0\end{array}\right.$, if $\sum_{i=1}^{n} a_{i j}=0, I_{j}=0$.

Normalize the coefficient of the operation sequence, then we can get the weight of operation sequence. 


$$
S_{q_{i}}=\frac{I_{j}}{\sum_{j=1}^{n} I_{j}}
$$

The weights of an operation sequence for components in tank command and control interface are in the table below.

The greater weight of the operating sequence means the component will be manipulated preferentially in the operation and the component should be positioned away from the center of the field of view. The weight of operation sequence for component $\mathrm{i}$ is denoted by $S q_{i}$, thus the sub-objective function of the operation sequence is obtained as follows:

$$
F_{3}(\boldsymbol{X}, \boldsymbol{Y})=\sum_{i=1}^{n} s_{q_{i}} \cdot d_{i p}
$$

where the larger value for $F_{3}(\boldsymbol{X}, \boldsymbol{Y})$ will represent a better layout conforming to the principle of operating sequence.

\section{The relevance}

The relevance of component is the degree to which two component information are related within a functional group. Relevance matrix is identified by the decimal less than or equal to 1 . The relevance of the component itself is 1 , which is shown in the table.

Components with higher relevance values should be placed in adjacent positions with each other in spatial locations, such as functionally relevant components should be placed in adjacent positions. The relevance coefficient between component $i$ and element $j$ is expressed as $R e_{i j}$, and the sub-objective function about the relevance of component is obtained as follows:

$$
F_{4}(\boldsymbol{X}, \quad \boldsymbol{Y})=\sum_{i=1}^{n} \sum_{j=i+1}^{n} \operatorname{Re}_{i j} \cdot\left(C-d_{i j}\right)
$$

Where the larger value for $F_{4}(\boldsymbol{X}, \boldsymbol{Y})$ will represent a better layout conforming to the principle of relevance.

\section{The uniformity}

The uniform layout of the interface has an important influence on the human-vehicle interaction for tank crew. The more uniform the components are in the interface layout, the higher the utilization of interface space will be. In this case, the operator will be more comfort in visual and accurate in operation. Therefore, the uniform layout of components is one of the principles that affect the optimal layout of the interface. The interface is equally divided into $m$ modules, the area of component $i$ is $S_{i}$, the area of component $i$ in the module $j$ is $S_{i j}$ and calculate the mean square error of the area occupied by all the components in each module. The smaller the value is, the more uniform the layout of the components will be. The corresponding objective function is as follows:

$$
\begin{gathered}
F_{5}(\boldsymbol{X}, \boldsymbol{Y})=\sqrt{\frac{\sum_{j=1}^{m}\left(\sum_{i=1}^{n} S_{i j}-A\right)^{2}}{m}} \\
A=\frac{\sum_{i=1}^{n} S_{i}}{m}
\end{gathered}
$$


where the larger value for $F_{5}(\boldsymbol{X}, \boldsymbol{Y})$ will represent a better layout conforming to the principle of uniformity.

\section{The operating path}

The layout of the human-vehicle interaction has a significant impact on the operating path of the tank crew in performing a particular mission. The shorter the operating path is, the higher the operational efficiency of the crew will be, as well as the stronger combat capability for the tank. Therefore, the operation path which crew chooses to complete the specific task is one of the important principles that affect the optimal layout of the interface. This paper aims at the tank to complete the target straight sight combat mission under the command and control interface, the operation path is shown as below:

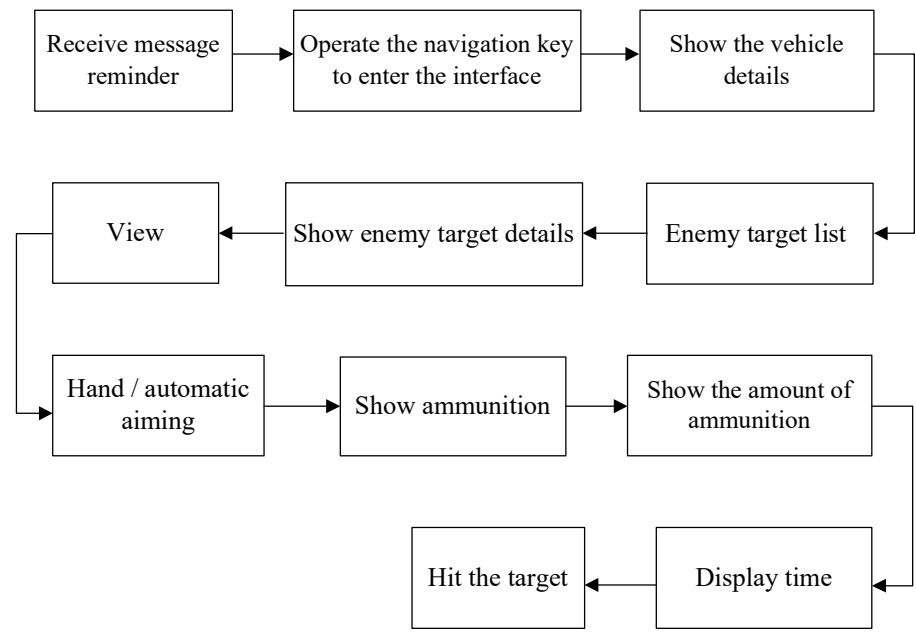

FIGURE 1. The operating path for a strike mission.

The distance between the component $i$ and the component $j$ is denoted by $d_{i j}$, thereby constructing the subobjective function of the operation path for the tank to complete the target straight sight combat mission:

$$
F_{6}(\boldsymbol{X}, \boldsymbol{Y})=d_{2,3}+d_{3,5}+d_{5,6}+d_{5,7}+d_{7,4}+d_{4,8}+d_{8,9}+d_{9,10}+d_{10,1}+d_{1,11}
$$

Where the smaller value for $F_{5}(\boldsymbol{X}, \boldsymbol{Y})$ will represent a better layout conforming to the principle of the shortest operating path.

\section{CONCLUSION}

In this study, we employed system engineering to quantitatively analyze the six layout principles of user interface, and take the frequency, importance, operation sequence, relevance, and consistency and operation path as evaluation indexes. Also we built a single objective function by weighted evaluation index. In the further study, we plan to optimize this problem with our model.

\section{REFERENCES}

1. Wang Ligang, Yuan Xiugan. Study on Computer Aided Design of Control Panel Based on Ergonomics[J]. Journal of Aeronautics, 1999,(S1):46-47.

2. Song Zhenghe, Mao Enrong, Zhou Yiming.Research on Optimal design Model of Human- Machine Interface in Mechanical Systerm[J]. Mechanical Design and Manufacturing, 2006, (02): 35-37.

3. Fu Shibo, Yuan Xiugan. The Knowledge System of optimization layout design in Work area.[J]. Journal of Beijing University of Aeronautics and Astronautics, 1997, (05): 127-130. 
4. Zeng Yi, Shen Lianwan, Qi Jiyang.Research on Particle Swarm Optimization Algorithm for Discrete Optimization of Equipment Layout[J]. Mechanical Design, 2006, (12): 4-6.

5. Yan Shengyuan, ,Zhang Jingling, Wang Shuaiqi. The Optimization of Manipulator based on multiple operating rules and principles.[J]. Journal of Shenyang University of Technology, 2012, 34(02): 169-173.

6. Wang Shanling.Study on Optimal Layout of Human Machine Interface Based on Genetic Algorithm[D]. Harbin Engineering University, 2009.

7. Chen Youhua, Zhao Jiabao. Research on the Compatibility of Fuzzy Judgment Matrix[J]. Operations and Management, 2004, (01): 44-47. 